\title{
The asymptotic profile of solutions of a class of singular parabolic equations.
}

\author{
F. Ragnedda, S. Vernier Piro and V. Vespri
}

\begin{abstract}
We consider a class of singular parabolic problems with Dirichlet boundary conditions. We use the Rayleigh quotient and recent Harnack estimates to derive estimates from above and from below for the solution. Moreover we study the asymptotic behaviour when the solution is approaching the extinction time.
\end{abstract}

Mathematics Subject Classification (2000). Primary 35K55; Secondary 35B40.

Keywords. Singular parabolic equation, asymptotic behaviour.

\section{Introduction}

Let $u(x, t)$ be the weak solution of the following initial boundary value problem

$$
\begin{gathered}
u_{t}=\operatorname{div} \mathbf{A}(x, t, \nabla u), \quad(x, t) \in \Omega \times(t>0), \\
u(x, t)=0, \quad(x, t) \in \partial \Omega \times(t>0), \\
u(x, 0)=u_{0}(x) \geq 0, \quad x \in \Omega,
\end{gathered}
$$

where $\Omega$ is a bounded domain in $\mathbb{R}^{N}$ with Lipschitz boundary, $u_{0} \in L^{1}(\Omega)$ and $\int_{\Omega} u_{0}(x) d x>0$. The functions $\mathbf{A}:=\left(A_{1}, \ldots, A_{N}\right)$ are regular and are assumed to satisfy the following structure conditions:

$$
\begin{aligned}
& \mathbf{A}(x, t, \nabla u) \nabla u \geq c_{0}|\nabla u|^{p}, \\
& |\mathbf{A}(x, t, \nabla u)| \leq c_{1}|\nabla u|^{p-1},
\end{aligned}
$$


with $\frac{2 N}{N+1}<p<2$ and $c_{0}, c_{1}$ given positive constants.

A function $u \in C_{l o c}\left(\mathbb{R}^{+} ; L_{l o c}^{2}(\Omega)\right) \cap L_{l o c}^{p}\left(\mathbb{R}^{+} ; W_{l o c}^{1, p}(\Omega)\right)$ is a weak solution of (1.1)(1.3), if for any compact subset $K$ of $\Omega$ and for every subinterval $\left[t_{1}, t_{2}\right] \in \mathbb{R}^{+}$

$$
\left.\int_{K} u \phi d x\right|_{t_{1}} ^{t_{2}}+\int_{t_{1}}^{t_{2}} \int_{K}\left(-u \phi_{t}+\mathbf{A}(x, t, \nabla u) \cdot \nabla \phi\right) d x d t=0,
$$

for all $\phi \in W_{l o c}^{1,2}\left(\mathbb{R}^{+} ; L^{2}(K)\right) \cap L_{l o c}^{p}\left(\mathbb{R}^{+} ; W_{0}^{1, p}(K)\right)$, where $\phi$ is a bounded testing function. We use this definition of solution because $u_{t}$ may have a modest degree of regularity and in general has meaning only in the sense of distributions. For more details see [2], Chap.II, Remark 1.1.

In the last few years, several papers were devoted to the study of the asymptotic behaviour of solutions to the porous media and the $p$-Laplace equations. We refer the reader to the recent monograph by Vazquez ([12]) and to the references therein. In almost all these references the Authors use elliptic results to study the asymptotic behaviour of the solutions. If, from one side, this makes the proof simple and very elegant, on the other hand it looks like this method is not flexible and cannot be applied for more general operators. In recent papers ([8]), ([9]) the Authors followed an alternative approach introduced by Berryman-Holland ([1]) and used in the context of the asymptotic behaviour of solutions to degenerate parabolic equations in [3], [7] and [10]. This approach is more parabolic than the previous one, namely, relying on the properties of the evolution equations, it is possible to study the asymptotic behaviour of the solutions and derive the elliptic properties of the asymptotic limit as a by-product. This new method is applied to study the case of equations with time dependent coefficients for the degenerate case.

We recall to the reader that in the singular case the phenomenon of the extinction of the solution in finite time occurs. This fact compells us to employ different techniques and mathematical tools with respect to the degenerate case. This generalization is based on recent techniques developed in [5], that allow us to avoid the use of comparison functions as in [3] and [10]. With respect the results proved in [8] and [9], here we use the Rayleigh quotient.

Remark 1.1. For the sake of simplicity, in this paper we consider only the case of initial boundary value problem (1.1)-(1.3), under the structure assumptions (1.4)-(1.5). It is possible to prove similar results in the case of porous-medium like equations, or even doubly nonlinear equations and for mixed boundary conditions (using the techniques introduced in [10]).

Remark 1.2. As we take the inital datum in $L^{1}(\Omega)$ we are compelled to limit ourselves to the case $\frac{2 N}{N+1}<p<2$. Actually, under this threshold, solutions of a Cauchy-Dirichlet problem with initial datum in $L^{1}$ could be unbounded (see, for instance, [2]).

The structure of the paper is as follows: in Section 2 we prove some preliminary results, that will be useful in the sequel. In Section 3, first we state some 
estimates from above, valid for the solution of the initial value problem (1.1)-(1.3) and then we prove proper estimates from below. In Section 4 we study the behaviour of solution up to the boundary. Finally in Section 5 we are able to prove the results concerning the asymptotic behaviour of the solution.

\section{Notation and preliminary results.}

We recall some notation and some results we will need to prove the main results.

Let $\mathcal{A}$ be a domain of $\mathbb{R}^{N}$ and let $|\mathcal{A}|$ denote the Lebesgue measure of the set $\mathcal{A}$. For $\rho>0$, let $B_{\rho}(x) \subset \mathbb{R}^{N}$ be the ball centered at $x$ of radius $\rho, B_{\rho}=B_{\rho}(0)$ and $\Omega_{\rho}(x)$ be equal to $B_{\rho}(x) \cap \Omega$.

We introduce the set

$$
Q_{\rho, \tau}\left(x_{0}, t_{0}\right):=B_{\rho}\left(x_{0}\right) \times\left(t_{0}, t_{0}+\tau\right),
$$

with $Q_{\rho, \tau} \subset \Omega \times(t>0)$ and

$$
\Omega_{\rho, \tau}\left(x_{0}, t_{0}\right):=\Omega_{\rho}\left(x_{0}\right) \times\left(t_{0}, t_{0}+\tau\right) .
$$

We recall now some results proved in [5] to which we refer the reader for the proof:

Theorem 2.1 ( $L_{l o c}^{1}-L_{l o c}^{\infty}$ Harnack-Type Estimates $)$. Let $u$ be a non-negative, weak solution to (1.1)-(1.5). Assume $p$ is in the super-critical range $\frac{2 N}{N+1}<p<2$. There exists a positive constant $\gamma$ depending only upon the data, such that for all cylinders

$$
\begin{gathered}
\Omega_{2 \rho}(y) \times[t, t+s] \\
\sup _{\Omega_{\rho}(y) \times\left[t+\frac{s}{2}, t+s\right]} u \leq \frac{\gamma}{(s)^{\frac{N}{\lambda}}}\left(\int_{\Omega_{2 \rho}(y)} u(x, t) d x\right)^{\frac{p}{\lambda}}+\gamma\left(\frac{s}{\rho^{p}}\right)^{\frac{1}{2-p}}
\end{gathered}
$$

where

$$
\lambda \stackrel{\text { def }}{=} N(p-2)+p
$$

Theorem 2.2 (An $L_{l o c}^{1} \quad$ Form of the Harnack Inequality for all $1<p<2$ ). Let $u$ be a non-negative, weak solution to (1.1)(1.5). Assume let $1<p<2$ and consider a ball $B_{\rho}(y)$ such that $B_{2 \rho}(y) \subset \Omega$. Then there exists a positive constant $\gamma$ depending only upon the data, such that for all cylinders $B_{2 \rho}(y) \times[s, t]$

$$
\sup _{s<\tau<t} \int_{B_{\rho}(y)} u(x, \tau) d x \leq \gamma \inf _{s<\tau<t} \int_{B_{2 \rho}(y)} u(x, \tau) d x+\gamma\left(\frac{t-s}{\rho^{\lambda}}\right)^{\frac{1}{2-p}}
$$

where $\lambda=N(p-2)+p$. The constant $\gamma=\gamma(p) \rightarrow \infty$ as either $p \rightarrow 2$ or as $p \rightarrow 1$. 
Theorem 2.3 (Intrinsic Harnack Estimate). Let u be a non-negative, weak solution to (1.1)-(1.5). Assume $p$ is in the super-critical range $p_{*}=\frac{2 N}{N+1} \leq p \leq 2$. There exist positive constants $\delta_{*}$ and $c$, depending only upon the data, such that for all $P_{o} \in \Omega \times(0, \infty)$ and all cylinders of the type $Q_{8 \rho}\left(P_{o}\right) \subset \Omega \times(0, \infty)$,

$$
c u\left(x_{o}, t_{o}\right) \leq \inf _{B_{\rho}\left(x_{o}\right)} u(\cdot, t)
$$

for all times

$$
t_{o}-\delta_{*}\left[u\left(P_{o}\right)\right]^{2-p} \rho^{p} \leq t \leq t_{o}+\delta_{*}\left[u\left(P_{o}\right)\right]^{2-p} \rho^{p} .
$$

The constants $c$ and $\delta_{*}$ tend to zero as either $p \rightarrow 2$ or as $p \rightarrow p_{*}$.

We point out that this inequality is simultaneously a "forward and backward in time" Harnack estimate as well as a Harnack estimate of elliptic type. Inequalities of this type would be false for non-negative solutions of the heat equation. This is reflected in (2.10)-(2.11), as the constants $c$ and $\delta_{*}$ tend to zero as $p \rightarrow 2$. As proved in [5] It turns out that these inequalities lose meaning also as $p$ tends to the critical value $p_{*}$

Remark 2.4. Let us stress that all the above results hold in the context of local nonnegative solutions of singular parabolic equations. For our purpose and for the sake of simplicity, we stated them only for non negative solutions of a CauchyDirichlet problem.

Let us quote now a well-known regularity result for singular parabolic equations (for the proof, see [2], Theorems 1.1 and 1.2, pages 77-78).

Theorem 2.5 (Regularity result). Let $u$ be a local weak solution to (1.1)-(1.3) in a domain $\Omega$ and assume that the structure conditions (1.4) and (1.5) hold. Assume $1<p<2$. Then for each closed set $K$ strictly contained in $\Omega, u$ is uniformly Hölder continuous in $K$ with the Hölder continuity constant that depends in a quantitative way on the data. The same result holds for a solution of a CauchyDirichlet problem. More precisely, assume that u be a local weak solution to (1.1) (1.5). Assume let $1<p<2$. Then for each $\varepsilon>0$, u is uniformly Hölder continuous in $\Omega \times(\varepsilon, \infty)$ with the constant of Hölder continuity that depends in a quantitative way on the data.

Remark 2.6. All the results previously stated hold in the case that the function $\mathbf{A}=\left(A_{1}, \ldots, A_{n}\right)$ is assumed to be only measurable. The following result requires the differentaibility of the coefficients.

We consider now some auxiliary results to be used later. First we introduce the Rayleigh quotient

$$
E(u(t))=\frac{\int_{\Omega} \mathbf{A}(x, t, \nabla u) \cdot \nabla u d x}{\left(\int_{\Omega} u^{2} d x\right)^{p / 2}} .
$$

In addition, set $\mathbf{s}(x, t):=\nabla u(x, t)$, that is, $s_{i}=u_{x_{i}}, i=1, \ldots, N$. 
Let us denote by $\mathbf{A}_{t}$ the derivatives of $\mathbf{A}(x, t, \mathbf{s})$ with respect $t$ and with

$$
\mathbf{A}_{\mathbf{s}}:=\frac{\partial\left(A_{1}, \ldots, A_{N}\right)}{\partial\left(u_{x_{1}}, \ldots, u_{x_{N}}\right)} .
$$

Now we prove the following

Theorem 2.7 (Rayleigh quotient). Let u be a non-negative, weak solution to (1.1)(1.3) and assume that the structure conditions (1.4) and (1.5) hold with $p$ in the supercritical range $p_{*}=\frac{2 N}{N+1}<p \leq 2$. Let $T^{*}$ be the extinction time. Assume

the functions $A_{i}(x, t, \nabla u)$ to be differentiable,

$$
\int_{\Omega} \mathbf{A}_{t}(x, t, \nabla u) \cdot \nabla u d x \leq 0
$$

and

$$
\begin{gathered}
\int_{\Omega} \operatorname{div}(\mathbf{A}(x, t, \nabla u)) \operatorname{div}\left(\mathbf{A}_{\mathbf{s}}(x, t, \nabla u) \nabla u\right) d x \\
\geq(p-1) \int_{\Omega}|\operatorname{div} \mathbf{A}(x, t, \nabla u)|^{2} d x
\end{gathered}
$$

Then

$$
\|u\|_{L^{2}} \leq\left[(2-p) E(u(0))\left(T^{*}-t\right)\right]^{\frac{1}{2-p}}
$$

Remark 2.8. Condition (2.10) is naturally verified by equations like the p-laplacean.

\section{Proof.}

Following [4], prop.13, we prove that $E(u(t))$ under restrictions (2.8)- (2.10) is nonincreasing in time.

From the equation (1.1) we have

$$
\frac{1}{2} \frac{d}{d t}\|u\|_{L^{2}}^{2}=-\int_{\Omega} \mathbf{A}(x, t, \nabla u) \cdot \nabla u d x .
$$

On the other hand, by applying the divergence theorem and Hölder inequality we get

$$
\int_{\Omega} \mathbf{A}(x, t, \nabla u) \cdot \nabla u d x=-\int_{\Omega} u \operatorname{div} \mathbf{A} d x \leq\|u\|_{L^{2}}\left(\int_{\Omega}|\operatorname{div} \mathbf{A}|^{2} d x\right)^{\frac{1}{2}},
$$

from which we obtain

$$
\int_{\Omega}|\operatorname{div} \mathbf{A}|^{2} d x \geq \frac{\left(\int_{\Omega} \mathbf{A}(x, t, \nabla u) \cdot \nabla u d x\right)^{2}}{\int_{\Omega} u^{2} d x} .
$$

Moreover, by using once more (1.1), we have

$$
\frac{d}{d t} \int_{\Omega} \mathbf{A}(x, t, \nabla u) \cdot \nabla u d x=-\int_{\Omega}|\operatorname{div} \mathbf{A}|^{2} d x+\int_{\Omega}\left(\mathbf{A}_{t} \nabla u+\mathbf{A}_{\mathbf{s}} \nabla u_{t} \nabla u\right) d x .
$$


By inserting (2.9) and (2.10) in (2.15) and then using (2.14) we obtain

$$
\frac{d}{d t} \int_{\Omega} \mathbf{A}(x, t, \nabla u) \cdot \nabla u d x \leq-p \int_{\Omega}|\operatorname{div} \mathbf{A}|^{2} d x \leq-p \frac{\left(\int_{\Omega} \mathbf{A} \cdot \nabla u d x\right)^{2}}{\int_{\Omega} u^{2} d x}
$$

From (2.12) and (2.16) we deduce

$$
\frac{\frac{d}{d t} \int_{\Omega} \mathbf{A}(x, t, \nabla u) \cdot \nabla u d x}{\int_{\Omega} \mathbf{A}(x, t, \nabla u) \cdot \nabla u d x} \leq-p \frac{\int_{\Omega} \mathbf{A}(x, t, \nabla u) \cdot \nabla u d x}{\int_{\Omega} u^{2} d x}=\frac{p}{2} \frac{\frac{d}{d t} \int_{\Omega} u^{2} d x}{\int_{\Omega} u^{2} d x}
$$

and (2.17) implies that $E(u(t))$ is nonincreasing in time. To prove (2.11) we remark that inserting the Rayleigh quotient in (2.12) we get

$$
\frac{d}{d t}\|u(t)\|_{2}^{2-p}=-(2-p) E(u(t))
$$

From (2.18) one easily deduces (2.11).

Remark 2.9. If we assume $\mathbf{A}(x, t, \nabla u)$ depending on $u$, Theo. 2.7 holds by replacing (2.10) with

$$
\int_{\Omega} \operatorname{div} \mathbf{A}(x, t, u, \nabla u)\left[\operatorname{div}\left(\mathbf{A}_{\mathbf{s}} \nabla u\right)-\mathbf{A}_{u}\right] d x \geq(p-1) \int_{\Omega}|\operatorname{div} \mathbf{A}|^{2} d x .
$$

\section{Estimate from above and below.}

We show in this section that the solution of $(1.1)-(1.3)$ is bounded both from above and from below under structure conditions (1.4)-(1.5) and (2.8)- (2.10).

\section{1. $L^{2}$-Estimate from below.}

In this section we prove the estimates from above, we will need in the proof of the main result.

Theorem 3.1. Let u be a non-negative, weak solution to (1.1)-(1.3). Assume (1.4), (1.5) and (2.8)- (2.10) hold. Assume $p$ is in the supercritical range $p_{*}=\frac{2 N}{N+1}<$ $p \leq 2$. Then there is a finite time $T^{*}$, depending only upon $N, p$ and $u_{0}$, such that $u(\cdot, t)=0$ for all $t \geq T^{*}$. Moreover there exists a constant $\gamma_{2}>0$ depending only upon $N$ and $p$ such that for each $0<t<T^{*}$

$$
\gamma_{2}\|u\|_{L^{2}}^{2-p}|\Omega|^{\frac{N(p-2)+2 p}{2 N}} \geq\left(T^{*}-t\right) .
$$

\section{Proof.}

We follow the same pattern of [4], [6] and [10], to which we refer for more details. First of all note that by Theorem 2.1 for each $t>0, u(\cdot, t)$ belongs to $L^{\infty}$ and its $L^{\infty}$-norm is controlled by the $L^{1}$-norm of the initial datum. As the measure of $\Omega$ is finite, we have that, for any $t>0, u(\cdot, t)$ belongs to $L^{2}$ and its norm is controlled by the $L^{1}$-norm of the initial datum. 
From the definition of weak solution, choosing as test function $\phi=u$ and integrating in the space variables, it follows that

$$
\frac{1}{2} \frac{d}{d t}\|u\|_{L^{2}}^{2}=-\int_{\Omega} \mathbf{A}(x, t, \nabla u) \cdot \nabla u d x .
$$

By the structure condition (1.4), we get

$$
\frac{1}{2} \frac{d}{d t}\|u\|_{L^{2}}^{2} \leq-c_{0} \int_{\Omega}|\nabla u|^{p} d x .
$$

By the Hölder and Sobolev inequalities

$$
\left(\int_{\Omega} u^{2} d x\right)^{\frac{p}{2}} \leq \gamma|\Omega|^{\frac{N(p-2)+2 p}{N}} \int_{\Omega}|\nabla u|^{p} d x,
$$

where the constant $\gamma$ does not depend upon $|\Omega|, p, N$. Plugging (3.4) into (3.3), we have

$$
\frac{1}{2} \frac{d}{d t}\|u\|_{L^{2}}^{2} \leq-\frac{1}{\gamma}|\Omega|^{\frac{N(p-2)+2 p}{N}}\left(\int_{\Omega} u^{2} d x\right)^{\frac{p}{2}} .
$$

For any $t>0$, the function $\psi:=\int_{\Omega} u^{2}(x, t) d x$ satisfies the following ordinary differential inequality

$$
\dot{\psi}+\frac{2}{\gamma|\Omega|^{\frac{N(p-2)+2 p}{N}}} \psi^{p / 2} \leq 0 .
$$

with $\psi(\varepsilon)<\infty$ for any $\varepsilon>0$ (the solution belongs to $L^{2}$ for any positive time). Therefore, by starting from a positive time and by integrating (3.6), one can deduce the existence of a finite extinction time $T^{*}$. In an analogous way, it is possible to prove (3.1) by the ordinary differential inequality (3.6).

\section{2. $L^{2}$-Estimates from above.}

In an almost straightforward way from Theorem 2.2 we have

Theorem 3.2. Let u be a non-negative, weak solution to (1.1)-(1.3). Assume (1.4), (1.5) and (2.8)-(2.10) hold. Assume $p$ is in the super-critical range $p_{*}=\frac{2 N}{N+1}<$ $p<2$. Then there is a constant $\gamma_{3}$ (depending only upon $N$ and $p$ ) such that, for any $t_{o}<T^{*}$

$$
\left(\int_{\Omega} u^{2}\left(x, t_{o}\right) d x\right)^{\frac{1}{2}} \leq \gamma_{3}\left(T^{*}-t_{o}\right)^{\frac{1}{2-p}}
$$

\section{Proof.}

Arguing as in [4], pag 72, we have that

$$
\frac{d}{d t}\|u\|_{L^{2}}^{2-p}=-(2-p) E(u(t)) .
$$

By Theorem 2.7 we have that $E(u(t))$ is non increasing in time and choosing a time $t_{0}$, we have for any $t_{0}<t<T^{*}$

$$
\|u\|_{L^{2}} \leq\left[(2-p) E\left(u\left(t_{0}\right)\right)\left(T^{*}-t_{0}\right)\right]^{\frac{1}{2-p}} .
$$


We need now an estimate of $E\left(u\left(t_{0}\right)\right)=\frac{\int_{\Omega} \mathbf{A} \cdot \nabla u d x}{\int_{\Omega} u^{2} d x}$ from above. By Theorem 3.1 and (1.5) we obtain

$$
E\left(u\left(t_{0}\right)\right) \leq \tilde{\gamma} \int_{\Omega}|\mathbf{A}(x, t, \nabla u) \cdot \nabla u| d x \leq \tilde{\gamma} c_{1} \int_{\Omega}|\nabla u|^{p} d x
$$

In order to get an estimate from above $\int_{\Omega}|\nabla u|^{p} d x$ we follow [8], Theorem 4.1, step 2.

By $L^{\infty}$ estimate (2.2), Theorem 2.1, we have that for any $t$ such that $\frac{T^{*}}{2}<t<T^{*}$, $u(t) \in L^{\infty}(\Omega)$ and then $u \in L^{2}(\Omega)$, since $\Omega$ is bounded. Now we have to prove that there exists a time $t_{0}$ such that $\int_{\Omega}|\nabla u|^{p} d x$ is bounded. Indeed starting from (1.6) with $\phi=u$, we get

$$
\frac{1}{2} \int_{\Omega} u^{2}\left(x, T^{*}\right) d x-\frac{1}{2} \int_{\Omega} u^{2}\left(x, \frac{T^{*}}{2}\right) d x \leq-c_{0} \int_{\frac{T^{*}}{2}}^{T^{*}} \int_{\Omega}|\nabla u|^{p} d x d t,
$$

which yields

$$
c_{0} \int_{\frac{T^{*}}{2}}^{T^{*}} \int_{\Omega}|\nabla u|^{p} d x d t \leq \frac{1}{2} \int_{\Omega} u^{2}\left(x, \frac{T^{*}}{2}\right) d x .
$$

This implies that there exists a time level $t_{0} \in\left[\frac{T^{*}}{2}, T^{*}\right]$ where

$$
c_{0} \int_{\Omega}|\nabla u|^{p} d x \leq \frac{1}{T^{*}} \int_{\Omega} u^{2}\left(x, \frac{T^{*}}{2}\right) d x .
$$

So we can estimate $E\left(u\left(t_{0}\right)\right)$ and therefore $\|u\|_{L^{2}}$.

\section{3. $\quad L^{\infty}$-Estimates from above.}

By Theorem 2.1 and by inequality (3.7) we get the $L^{\infty}$-estimates from above.

Theorem 3.3. Let $u$ be a non-negative, weak solution to (1.1)-(1.3) Assume (1.4), (1.5) and (2.8)- (2.10) hold. Assume $p$ is in the super-critical range $p_{*}=\frac{2 N}{N+1}<$ $p<2$. Then there is a constant $\gamma_{4}$ (depending only upon $N$ and $p$ ) such that, for any $t_{o}<T^{*}$

$$
\sup _{\Omega} u\left(x, t_{o}\right) \leq \gamma_{4}\left(T^{*}-t_{o}\right)^{\frac{1}{2-p}}
$$

\section{Proof.}

Inequality (3.14) follows from (2.2) choosing $t=T^{*}-2 t_{o}, t+s=T^{*}, \rho=d_{\Omega}$, where $d_{\Omega}$ be the diameter of $\Omega$ and estimating $\int_{\Omega} u\left(x, t_{o}\right) d x$ with $\gamma\left(T^{*}-t_{o}\right)^{\frac{1}{2-p}}$ by using (3.7). 


\section{4. $L^{\infty}$ interior estimates from below.}

From Theorems 3.1 and 3.3 we can deduce estimates in the interior of $\Omega$. More precisely

Theorem 3.4. Let $u$ be a non-negative, weak solution to (1.1)-(1.3). Assume (1.4), (1.5) and (2.8)-(2.10) hold. Assume $p$ is in the super-critical range $p_{*}=\frac{2 N}{N+1}<$ $p<2$. There exists a positive number $d$, depending only upon the geometry of $\Omega, p$ and $N$, such that for any $\frac{T^{*}}{2}<t_{o}<T^{*}$, there is a point $x_{o} \in \Omega$ with $\operatorname{dist}\left(x_{o}, \partial \Omega\right)>d$ such that

$$
u\left(x_{o}, t_{o}\right) \geq \gamma_{5}\left(T^{*}-t_{o}\right)^{\frac{1}{2-p}},
$$

where $\gamma_{5}$ is a positive constant depending only upon the data.

\section{Proof.}

By (3.1) we have

$$
\sup _{\Omega} u\left(x, t_{o}\right) \geq|\Omega|^{-\frac{1}{2}}|| u \|_{L^{2}} \geq \gamma_{6}\left(T^{*}-t_{o}\right)^{\frac{1}{(2-p)}}
$$

and then by (3.14)

$$
\|u\|_{L^{2}} \geq \gamma_{7} \sup _{\Omega} u\left(x, t_{o}\right)
$$

with $\gamma_{7}=\gamma_{7}(n, p, \Omega)$.

Define the set $\mathrm{A}$ as the set of the points $x \in \Omega$ where $u\left(x, t_{o}\right) \geq \frac{\gamma_{7}}{\sqrt{2|\Omega|}} \sup _{\Omega} u\left(x, t_{o}\right)$.

Let $\gamma_{8}=\frac{\gamma_{7}}{\sqrt{2|\Omega|}} \sup _{\Omega} u\left(x, t_{o}\right)$.

By (3.17) we have

$$
|\mathrm{A}| \geq \frac{1}{2} \gamma_{7}^{2}
$$

Since the set $A$ has strictly positive measure and $\Omega$ is a bounded regular set, then there exists a positive constant $d$ such that exists a point $x_{o} \in \mathrm{A}$ with $\operatorname{dist}\left(x_{o}, \mathrm{~A}\right)>d$. Then (3.15) follows from the definition of $\gamma_{8}$ and (3.16).

The following statement is a direct consequence of Theo. 3.4 and of the intrinsic Harnack estimates of Theorem 2.3.

Theorem 3.5. Let $u$ be a non-negative, weak solution to (1.1)-(1.3). Assume (1.4), (1.5) and (2.8) - (2.10) hold. Assume that $p$ is in the super-critical range $p_{*}=$ $\frac{2 N}{N+1}<p<2$. For any positive number $d$ there is a constant $\gamma_{9}$ depending only upon the geometry of $\Omega, d, p$ and $N$, such that, for any $\frac{T^{*}}{2}<t_{o}<T^{*}$, for any point $x_{o} \in \Omega$ with $\operatorname{dist}\left(x_{o}, \partial \Omega\right)>d$

$$
u\left(x_{o}, t_{o}\right) \geq \gamma_{9}\left(T^{*}-t_{o}\right)^{\frac{1}{2-p}} .
$$




\section{Estimates at the boundary from above and from below}

Thanks to the previous estimates, we can extend some results up to the boundary. Let $d(x)$ be the distance from the point $x$ to the boundary $\partial \Omega$.

Theorem 4.1. Let $u$ be a non-negative, weak solution to (1.1)-(1.3). Assume (1.4), (1.5) and (2.8)- (2.10) hold. Assume $p$ is in the super-critical range $p_{*}=\frac{2 N}{N+1} \leq$ $p \leq 2$. There exist two constants $\beta \in(0,1)$ and $\gamma_{10}$, such that for each $x \in \Omega$ and for each $\frac{T^{*}}{2}<t<T^{*}$

$$
u(x, t) \geq\left(T^{*}-t\right)^{\frac{1}{2-p}} \gamma_{10} d(x)^{\beta}
$$

\section{Proof .}

Denote with $K_{n}=\left\{x \in \Omega\right.$ such that $\left.d(x) \geq 2^{-n}\right\}$. As the boundary is Lipschitzcontinuous, there exists $n_{0}$ such that for each $n \geq n_{0}$ the distance between $K_{n}$ and any point $x \in K_{n+1}$ is less or equal to $2^{-(n+1)}$. By Theorem 3.5 for any $x_{o} \in K_{n_{o}}$ and for any $\frac{T^{*}}{2}<t<T^{*}$

$$
u\left(x_{o}, t\right) \geq \gamma_{9}\left(T^{*}-t\right)^{\frac{1}{2-p}}
$$

By Theorem 2.3 we have that for each $x \in K_{n_{0}+1}$,

$$
u(x, t) \geq c u\left(x_{o}, t\right) \geq c \gamma_{9}\left(T^{*}-t\right)^{\frac{1}{2-p}} .
$$

By induction for any $x \in K_{n_{0}+n}$ one gets that

$$
u(x, t) \geq c^{n} \gamma_{9}\left(T^{*}-t\right)^{\frac{1}{2-p}} .
$$

As we are working with $2^{-n-1} \leq d(x) \leq 2^{-n}$, inequality (4.3) easily implies the statement of the Theorem.

Theorem 4.2. Let $u$ be a non-negative, weak solution to (1.1)-(1.3). Assume (1.4), (1.5) and (2.8)- (2.10) hold. Assume that $p$ is in the super-critical range $p_{*}=$ $\frac{2 N}{N+1} \leq p \leq 2$. There exist two constants $\eta \in(0,1)$ and $\gamma$, such that for each $x \in \Omega$ and for each $\frac{T^{*}}{2}<t<T^{*}$

$$
\gamma d(x)^{\eta} \geq u(x, t)\left(T^{*}-t\right)^{\frac{1}{2-p}}
$$

\section{Proof.}

Let $x_{o}$ be a point of $\partial \Omega$. As the boundary is Lipschitz continuous, there is a diffeomorphism bi-Lipshcitz continuous $\mathcal{T}$ that maps a neighborhood of $x_{o}$ in the hemishpere $B^{+}(0,1)=\left\{x \in B(0,1)\right.$ such that $\left.x_{N}>0\right\}$. The function $v(y, t)=$ $u\left(\mathcal{T}^{-1} y, t\right)$ is a solution of

$$
\begin{gathered}
v_{t}=\operatorname{div} \mathbf{A}_{1}(y, t, \nabla v), \quad(y, t) \in B^{+}(0,1) \times(t>0), \\
v(y, t)=0, \quad t>0 \quad y \in B(0,1) \cap\left\{y \in \mathbb{R}^{N} \text { such that } y_{N}=0\right\},
\end{gathered}
$$

where

$$
\mathbf{A}_{\mathbf{1}}(y, t, \nabla v)=\mathbf{A}\left(\mathcal{T}^{-1} y, t, J\left(\mathcal{T}^{-1}\right)(y) \nabla u\left(\mathcal{T}^{-1} y, t\right)\left|J\left(\mathcal{T}^{-1}\right)(y)\right|\right)
$$


$J\left(\mathcal{T}^{-1}\right)(y)$ is the Jacobian matrix and $\left|J\left(\mathcal{T}^{-1}\right)(y)\right|$ is the Jacobian determinant. Let us extend $v$ in $B(0,1)$. Denote with $\bar{y}$ the first $N-1$ components of the vector $y$. Define $w(y, t)=v(y, t)$ if $y_{n} \geq 0$ and $\left.w(y, t)=-v\left(\bar{y},-y_{N}\right), t\right)$ if $y_{n} \leq 0$. The function $w$ is a solution of

$$
w_{t}=\operatorname{div} \mathbf{A}_{\mathbf{2}}(y, t, \nabla w), \quad(w, t) \in B(0,1) \times(t>0),
$$

where $\mathbf{A}_{\mathbf{2}}$ is equal to $\mathbf{A}_{\mathbf{1}}$ if $y_{N} \geq 0$; when $y_{N} \leq 0$

$$
\begin{gathered}
\mathbf{A}_{\mathbf{2}}\left(y ; t ; D_{1} w, \cdots, D_{N-1} w, D_{N} w\right)= \\
-\mathbf{A}_{2}\left(\left(\bar{y},-y_{N}\right) ; t, D_{1} w\left(\left(\bar{y},-y_{N}\right), t\right), \cdots, D_{N-1} w\left(\left(\bar{y},-y_{N}\right), t\right),\right. \\
\left.-D_{N} w\left(\left(\bar{y},-y_{N}\right), t\right)\right)
\end{gathered}
$$

As $\mathbf{A}_{2}$ satisfies the structure conditions (1.4)-(1.5) by Theorem 2.5, the function $w$ is Hölder continuous and this implies (4.4).

\section{Asymptotic behaviour.}

In this section we investigate the behavior of the solution of (1.1)-(1.3) when it is approaching the extinction time. We work as in [3], [10] and [4] and we set $t=T^{*}-T^{*} e^{-\tau}$ and

$$
w(x, \tau)=\frac{u\left(x, T^{*}-T^{*} e^{-\tau}\right)}{\left(T^{*} e^{-\tau}\right)^{\frac{1}{2-p}}} .
$$

The function $w(x, \tau)$ is a non negative solution of the equation

$$
w_{\tau}=\operatorname{div} \tilde{\mathbf{A}}(x, \tau, \nabla w)+\frac{1}{2-p} w
$$

where

$$
\tilde{\mathbf{A}}(x, \tau, \nabla w):=\left(T^{*} e^{-\tau}\right)^{-\frac{p-1}{2-p}} \mathbf{A}\left(x, T^{*}-T^{*} e^{-\tau},\left(T^{*} e^{-\tau}\right)^{\frac{1}{2-p}} \nabla w\right)
$$

and

$$
w(x, 0)=u_{0}(x)\left(T^{*}\right)^{-\frac{1}{2-p}} .
$$

Note that $\tilde{\mathbf{A}}$ satisfies the structure conditions (1.4) - (1.5). From the results of the previous section we have:

Theorem 5.1. For any positive time $t_{1}$, for any closed set $K$ strictly contained in $\Omega$ there are strictly positive constants $C_{1}-C_{5}$, depending only upon the data, $t_{1}$ and $K$, such that for any $t \geq t_{1}$

- for any $x \in \Omega$

$$
w(x, t) \leq C_{1}
$$

- for any $x \in K$

$$
w(x, t) \geq C_{2}
$$


- for any $x \in \Omega$,

$$
C_{3} d(x)^{\beta} \leq w(x, t) \leq C_{4} d(x)^{\eta},
$$

where $d(x)$ the distance from the point $x$ to the boundary $\partial \Omega$.

- $w$ is uniformly $\alpha$-Hölder continuous with the Hölder continuity constant that depends only upon the data and

$$
\|w\|_{C^{\alpha, \frac{\alpha}{p}}\left(\Omega,\left[t_{1}, \infty\right]\right)} \leq C_{5} .
$$

By Theorem 5.1, $w(t)$ is equi-Hölder continuous, therefore, up to a subsequence, there is a function $v \in C^{\alpha}(\Omega)$ such that $w \rightarrow v$ in $C^{\alpha}$.

If we want the function $v$ to be the solution of a suitable partial differential equation, we have to assume some hypotheses on the coefficient $\tilde{\mathbf{A}}(x, \tau, \tilde{\mathbf{s}})$ in $(5.2)$, where $\tilde{\mathbf{s}}(x, t):=\nabla w(x, t)$, that is, $\tilde{s}_{i}=w_{x_{i}}, i=1, \ldots, N$.

$\tilde{\mathbf{A}}(x, \tau, \tilde{\mathbf{s}})$ is a $C^{0}$ function with respect the time,

$\exists$ a function $H(x, \tau, \tilde{\mathbf{s}})$ such that $\frac{\partial H}{\partial \tilde{s}_{i}}=\tilde{A}_{i}$,

$$
\text { and } \frac{\partial H}{\partial \tau} \leq 0
$$

$\exists \quad$ a positive constant $C_{6}: \int_{\Omega} H(x, \tau, \nabla w) d x \geq C_{6}$.

Note that the assumption of continuity on $\mathbf{A}(x, t, \mathbf{s})$ implies that

$$
\exists \lim _{\tau \rightarrow+\infty} \tilde{\mathbf{A}}(x, \tau, \tilde{\mathbf{s}})=\mathbf{A}_{\infty}(x, \tilde{\mathbf{s}}) .
$$

Theorem 5.2. Assume that hypotheses (1.4) and (1.5) hold. Then the function $v$ belongs to $W_{0}^{1, p} \cap L^{2}(\Omega)$ and it is a non trivial solution of

$$
\operatorname{div}\left(\mathbf{A}_{\infty}(x, \nabla v)\right)=-\frac{1}{2-p} v .
$$

\section{Proof.}

The functional

$$
F(x, \tau, \nabla w(x, \tau))=\int_{\Omega} H(x, \tau, \nabla w) d x-\frac{1}{2(2-p)} \int_{\Omega} w^{2}(x, \tau) d x
$$

is monotone decreasing in time. In fact

$$
\begin{aligned}
& \frac{d}{d \tau} \int_{\Omega} H d x=\int_{\Omega}\left[\frac{\partial H}{\partial \tilde{s}_{i}} \nabla_{i} w_{\tau}+\frac{\partial H}{\partial \tau}\right] d x \\
& \leq \int_{\Omega} \frac{\partial H}{\partial \tilde{s}_{i}} \nabla_{i} w_{\tau} d x=-\int_{\Omega}(\operatorname{div} \tilde{\mathbf{A}}) w_{\tau} d x \\
& =-\int_{\Omega}\left(w_{\tau}\right)^{2} d x+\frac{1}{2-p} \int_{\Omega} w w_{\tau} d x .
\end{aligned}
$$


Then

$$
\frac{d F}{d \tau} \leq-\int_{\Omega}\left(w_{\tau}\right)^{2} \leq 0
$$

As the functional $F$ is bounded from below, this implies that, up to a subsequence, $\frac{d F}{d \tau}$ (and therefore $\left.\int_{\Omega} w_{\tau}^{2}(x) d x\right)$ converges to zero.

Then $w$ converges to its limit $v \in W_{0}^{1, p} \cap L^{2}(\Omega)$ and $v$ is the solution of (5.11).

\section{References}

[1] J.G. Berryman, C.J. Holland, Stability of the separable solution for fast diffusion Arch. Rational Mech. Anal. 74 (1980), no. 4, 379-388.

[2] E. Di Benedetto, Degenerate parabolic equations, Springer Verlag, (1993)

[3] E. Di Benedetto, Y. C. Kwong, V. Vespri, Local space analiticity of solutions of certain parabolic equations Indiana Univ. Math. J. 40 (1991), 741-765.

[4] E. Di Benedetto, J.M.Urbano, V.Vespri,Current Issues on Singular and Degenerate Evolution Equations Evolutionary equations. Vol.1, Handb. Differ. Equ., NorthHolland, Amsterdam, (2004), 169-286.

[5] E. Di Benedetto, U. Gianazza, V. Vespri, Forward, Backward and Elliptic Harnack Inequalities for Non-Negative Solutions to Certain Singular Parabolic Partial Differential Equations Annali Scuola Nor. Sup., IX, issue 2, (2010), 385-422.

[6] U. Gianazza, V. Vespri, A Harnack Inequality for a Degenerate Parabolic Equation, Journal of Evolution Equations 6 (2006), 247-267.

[7] J.J. Manfredi, V. Vespri, Large time behaviour of solutions to a class of Doubly Nonlinear Parabolic equations Electronic J. Diff. Eq. 2 (1994), 1-17.

[8] F. Ragnedda, S. Vernier Piro, V. Vespri, Large time behaviour of solutions to a class of nonautonomus degenerate parabolic equations Math. Annalen, DOI : 10.1007/s00208010-0496-4.

[9] F. Ragnedda, S. Vernier Piro, V. Vespri, Asymptotic time behaviour for nonautonomous degenerate parabolic problems with forcing term Nonlinear Anal. 71 (2009), e2316-e2321.

[10] G. Savaré, V. Vespri, The asymptotic profile of solutions of a class of doubly nonlinear equations Nonlinear Anal. 22 (1994), no. 12, 1553-1565.

[11] J.L. Vázquez, Asymptotic behaviour and propagation properties of the onedimensional flow of gas in a porous medium Trans. Amer. Math. Soc., 277 (1983), no. $2,507-527$.

[12] J.L. Vázquez, Smoothing and decay estimates for nonlinear diffusion equations. Equations of porous medium type Oxford Lecture Series in Mathematics and its Applications, 33, Oxford University Press, Oxford, 2006. 


\section{F. Ragnedda}

Dipartimento di Matematica e Informatica,

Viale Merello 92

09123 Cagliari

Italy

e-mail: ragnedda@unica.it

S. Vernier Piro

Dipartimento di Matematica e Informatica

Viale Merello 92

09123 Cagliari

Italy

e-mail: svernier@unica.it

V. Vespri

Dipartimento di Matematica "U. Dini", viale Morgagni 67/a

50134 Firenze

Italy

e-mail: vespri@math.unifi.it 